Rapid Reviews COVID-19

\title{
Review 2: "A Sew-Free Origami Mask for Improvised Respiratory Protection"
}

\section{Peter Germonpre ${ }^{1}$}

${ }^{1}$ Military Hospital Brussels, Centre for Hyperbaric Oxygen Therapy, Belgium

Published on: Nov 06, 2020

DOI: $10.1162 / 2$ e3983f5.a8ce9502

License: Creative Commons Attribution 4.0 International License (CC-BY 4.0). 


\section{$\underline{\text { RR:C19 Evidence Scale rating by reviewer: }}$}

- Potentially informative. The main claims made are not strongly justified by the methods and data, but may yield some insight. The results and conclusions of the study may resemble those from the hypothetical ideal study, but there is substantial room for doubt. Decision-makers should consider this evidence only with a thorough understanding of its weaknesses, alongside other evidence and theory. Decisionmakers should not consider this actionable, unless the weaknesses are clearly understood and there is other theory and evidence to further support it.

******************************************

\section{Review:}

The paper describes a simple "origami" folding design for a respiratory mask to be made out of filtration material, and claims that this would provide better respiratory prevention than currently proposed cloth masks while being much less costly and easier to make. They claim that using this design would be likely to promote greater mask-wearing tolerance and acceptance.

The authors further report the results of quantitative fit testing of the mask fabricated with different materials, and compare these with one surgical mask and one KN95 mask, locally sourced. They have not tested $\mathrm{CO} 2$ retention using the different materials used.

The method for fabricating the mask is adequately described and needs only minor modifications (such as how to measure the adequate length of the elastic bands prior to stapling). The obtained mask indeed seems to fit the average face shape well, provided sufficient tension is applied with the elastic bands. Re-positioning the bands and stapling multiple times will obviously reduce the filtration efficiency of the mask by creating additional holes.

As to the efficiency of the mask, the testing has exclusively taken place on a mannequin head, human subjects having only been used to obtain a "comparison" Fit Factor to verify the results obtained with the author's setup using a "environment chamber" containing the mannequin head and a scanning mobility particle sizer (SPMS) comparing the number of $\mathrm{NaCl}$ particles between environment and mannequin inhalation stream. Why they did not use the validated Quantitative Fit Testing procedure (OSHA Appendix A to §1910.134, which used human test subjects), is not 
clear. The differences reported between "optimal fit" (with the mask duct-taped to the mannequin head) and "nominal fit" (without additional sealing) may be interesting but are not relevant to human use: "optimal fit" should correspond to the actual filtration capacity of the material used (minus the holes created by the staples) whereas "nominal fit" on a hard-plastic mannequin face would obviously be different from a mask fitted on a human face. Also, using their testing chamber setup, pressure drop and flow resistance may be measured across the material, but only at the fixed "inhalation" flow of $60 \mathrm{Lpm}$, which is completely different from "real-life" conditions, with inspiration, expiration and a variable flow. This at best compares the properties of the material and not of the mask as a whole. In summary, additional to the "bench" testing of the mask (flow resistance, pressure drop, filtration properties of the materials used; all very interesting data), the masks should have been tested using a full Fit Testing with the Portacount Pro+ device using the OSHA protocol.

RR:C19 Strength of Evidence Scale - "Potentially Informative": While correctly claiming that the quality of filtration material and mask design (mask fit) are important factors in the degree of protection offered, the claims that the origami mask provides greater comfort compared to commercial alternatives, leading to promoting greater mask tolerance and acceptance, is not tested or verified. Furthermore, the claim that the mask performs better than KN95 masks is not substantiated, as that depends on a) the filtration material(s) used; b) the possible errors in fabrication of the mask (e.g. elastic loops to long, multiple staple holes) and c) the fitting of the mask on the face (e.g. taking into account facial hair or beard).

Therefore, I recommend the paper should be either significantly revised (Major Revision) or separated into two papers, one describing the mask design and comparing this with existing KN95 masks (e.g. creating such a mask from the same fabric as KN95 masks approved for medical use) and the other comparing the filtration and resistance/pressure drop properties of the various commercially available fabric and materials as reported now (but not necessarily in mask form-unless human subject OSHA Fit Testing is performed). 